\title{
Acción pública organizada y los procesos de gobierno a nivel local
}

\section{Organized public action and government processes at local level}

José Santos Zavala*

El Colegio de San Luis, San Luis Potosí, México

ISSN: ISSN-OI85-4259; e- ISSN: 2007-9I76 DoI: http://dx.doi.org/I0.28928/ri/7720I4/aotI/santoszavalaj

\section{Resumen}

En este artículo se busca definir los conceptos básicos y los niveles de análisis que permitan, desde una perspectiva organizacional, el estudio de los procesos de gobierno a nivel local, cuyo funcionamiento se explica a partir del supuesto de que es resultado de las acciones de los miembros de diversas organizaciones públicas de los tres niveles de gobierno. Este proceso de interacción es producto de las transformaciones sociales, de una mayor competencia electoral a nivel local, así como de la descentralización del gasto público federal.

Palabras claveः gobierno local, políticas publicas locales, decisión pública, instituciones, estrategia

\section{Abstract}

This article seeks to define the basic concepts and levels of analysis must be, from an organizational perspective, the study of the processes of governance at the local level. The operation is explained on the assumption that results from the actions of members of various public organizations in the three levels of government. This process of interaction is the product of social transformations, greater electoral competition locally, and the decentralization of federal government spending. Key words: local government, local public policy, public decision, institutions, strategy

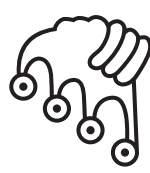

\section{IZTAPALAPA}

Agua sobre lajas

* Doctor en Estudios Organizacionales, por la Universidad Autónoma Metropolitana Unidad Iztapala y École des Hautes Études Commerciales de Montreal. Adscrito a El Colegio de San Luis, A. C. jszslp@gmail.com 

a solución de los problemas públicos locales es un proceso dinámico, complejo y conflictivo, motivo por el cual su análisis debe abordarse desde un enfoque multidisciplinario, tanto teórico como metodológico; su estudio ha de hacerse a partir de la reconstrucción de las acciones de los actores implantadas en un contexto institucional específico, los conceptos centrales serían: acción, actor, institución y estrategia, siendo las principales variables explicativas de las políticas públicas implementadas a nivel local.

El conjunto de acciones de las organizaciones públicas locales que intervienen en la solución de los problemas públicos locales conforman un sistema de acción pública que funciona con múltiples mecanismos de regulación (Cabrero, 2005); es configurada por una estructura institucional concreta y por un sistema de poder local específico. Con base en esto, se plantea el tránsito conceptual de los procesos de gobierno cimentados en la racionalidad-acción al de la complejidad-subjetividad-acción, la cual es más útil para entenderlos como un proceso dinámico, complejo y conflictivo.

El objetivo de este artículo es definir los conceptos básicos y los niveles de análisis que permitan, desde una perspectiva organizacional, el estudio de los procesos de gobierno a nivel local, cuyo funcionamiento se explica a partir del supuesto de que es resultado de las acciones de los actores de diversas organizaciones públicas de los tres niveles de gobierno. Este proceso de interacción es producto de las transformaciones sociales, de una mayor competencia electoral a nivel local, así como de la descentralización del gasto público federal.

Para plantear el tránsito del análisis de la organización al de la acción organizada en los procesos de solución de los problemas públicos a nivel local, este artículo se ha dividido en dos apartados; en el primero se establece el marco conceptual de la acción organizada a nivel local; en el segundo se define la estrategia como principal variable explicativa del funcionamiento del sistema de acción organizada. Con este marco conceptual se pretende contribuir al debate del análisis de los problemas públicos en el ámbito local 


\section{Los procesos de gobierno como un sistema de acción pública organizada}

Considerar los estudios organizacionales (во) como marco conceptual para el estudio de un conjunto de organizaciones públicas en interacción compleja y conflictiva implica adoptar un enfoque que abandone la idea de que el gobierno funciona con base en organizaciones aisladas, monolíticas, racionales y homogéneas (Arellano, Cabrero, Del Castillo, 2000); por el contrario, significa considerar que es un proceso social complejo y conflictivo en constante interacción y transformación.

En el análisis de los procesos de gobierno, desde una perspectiva organizacional, se parte del supuesto de que en la resolución de los problemas públicos participan diversas organizaciones públicas que interactúan de manera conflictiva en acciones específicas. Esto significa que los procesos son producto de un conjunto organizacional y no solo de una organización, que son configurados por actores que responden a diversas lógicas de acción, por lo que sus acciones no son totalmente racionales, sino subjetivas y producto de una estructura de poder local.

$\mathrm{Al}$ conceptualizar los procesos de gobierno a nivel local como resultado de las acciones de un conjunto de organizaciones se genera la idea de una compleja red de interacción de decisiones y acciones que generalmente son producto de una doble lógica de acción: la política y la administrativa. Sin embargo, en experiencias concretas de solución de problemas públicos en el ámbito local, una de ellas domina, lo cual es resultado del tipo y la forma de definición del problema, así como de la estructura de poder que lo rodea.

La lógica política se refiere a las percepciones y los intereses de los individuos y los grupos locales, que se manifiestan en la forma de definir los problemas públicos; representa la parte subjetiva de las acciones de los actores de las políticas públicas. La lógica administrativa considera que el actor actúa en función del campo de su competencia técnica, por lo que sus acciones responden a un proceso racional y documentado; el comportamiento se apega a las normas formales de la organización a la que pertenecen.

Al conceptualizar el proceso de gobierno como resultado de una doble lógica de acción, cada actor involucrado en la solución de los asuntos públicos tiene un comportamiento complejo, dinámico y conflictivo que, por un lado, debe responder a las necesidades de la población objetivo de las políticas públicas, pero a la vez debe considerar su dimensión organizacional interna, es decir, la estructura funcional, las normas y políticas, los grupos y situaciones coyunturales de funcionamiento de las organizaciones públicas. 
Para el estudio de las políticas públicas locales a partir de la acción organizada es necesario considerar a los actores que toman las decisiones, quienes, para ello, requieren recursos de acción, por lo que no son vistos como individuos, sino como parte de la estructura funcional de la organización en la que ocupan una posición, la cual les otorga recursos para establecer cierta dirección a las soluciones de los problemas públicos. Estos tomadores de decisiones consideran factores de orden técnico, político y de valores (Merino, 2013).

El concepto de acción organizada pone énfasis en el análisis de las interacciones de los actores, por lo que el conflicto y el poder son su principal fuente de explicación (Friedberg, 1993); las interacciones de los diversos actores se generan en situaciones organizacionales concretas, por lo cual su entendimiento se basa en la parcialidad que representan sus acciones del todo organizacional, en la naturaleza de las interacciones y en el sentido que les dan los diversos actores.

Este enfoque conceptual pone en el centro del análisis las acciones y sus actores, considerando al entorno institucional donde se generan, lo cual requiere una reflexión conceptual del tránsito del estudio de la organización al de un conjunto organizacional; pasar del reconocimiento de actor racional al de actor complejo y con intereses políticos; reconocer que la organización no funciona con base en la adaptación de la organización, sino que es producto de una relación de interdependencia con su entorno institucional, y que las acciones son construcciones sociales dinámicos.

\section{De la organización al análisis de los procesos de gobierno}

Diversos enfoques de los EO, para distinguirse y presentarse como alternativa teórica de análisis de la realidad social, han utilizado un concepto muy formalizado de organización, la cual han definido como una agrupación humana con carácter intencional, explícito y codificado de sus estructuras, funciones, procedimientos y objetivos (Friedberg, 1993); esto les ha permitido convertirse en una opción conceptual para entender los procesos de solución de los problemas de la sociedad.

Un enfoque racional de la organización, para el análisis de los procesos de interacción entre actores, revela poco los hechos reales de solución de los problemas públicos, ya que este va mucho más allá de los acuerdos formales de política pública; en su funcionamiento cotidiano los procesos de gobierno en organizaciones públicas incorporan objetivos y dinámica de las estructuras de poder local, en donde es posi- 
ble identificar las causas por las que una misma política pública obtiene diferentes resultados de un entorno institucional a otro.

Uno de los primeros enfoques de los eo que buscó superar las limitaciones del racional de organización fue el de March y Simon, quienes buscaron generar una visión más integral de todas aquellas aportaciones teóricas que, con excepción de la escuela de las relaciones humanas, se habían desarrollado de manera aislada del entorno; su principal contribución fue su propuesta conceptual sobre la racionalidad limitada de los actores dentro de las organizaciones.

Para analizar los procesos de gobierno como producto de un conjunto organizacional, es necesario considerarlo como un sistema de toma de decisiones; por lo que hay que prestar atención a las interfaces en que se generan las decisiones, las cuales se convierten en la base de control y desarrollo de las acciones de política pública. Así, las distintas organizaciones gubernamentales pueden ser consideradas como redes de comunicación, o bien como una jerarquía de redes de comunicación.

Para el análisis de los procesos de gobierno en la solución de los problemas públicos a nivel local, las aportaciones de March y Simon son de gran utilidad, principalmente en lo que respecta a la recuperación de los efectos de la estructura formal en el comportamiento de los actores al momento de tomar decisiones. Otra aportación relevante es su visión integradora del análisis de las organizaciones, que buscó dar cuenta de los aspectos racionales e irracionales del comportamiento de los actores.

Utilizar el concepto formal de organización que ha dominado en la mayor parte de los estudios de la burocracia puede ser útil en un contexto estable, donde hay dominio y sujeción de los actores, capitalización del saber, transparencia y previsibilidad e inexistencia de la competencia (Friedberg, 1993). Sin embargo, para una realidad local dinámica, compleja, de racionalidad limitada y de competencia política, como es el caso mexicano, la descripción de su estructura y sus reglas es solo una aproximación a su verdadero funcionamiento, dado que no se toma en cuenta la complejidad de los actores, sus valores, el poder y el conflicto como constructores de los procesos de gobierno.

Para este planteamiento es necesario reconocer los procesos de gobierno no como un todo organizacional monolítico, regido únicamente por estructuras formales, que se adaptan al contexto y que funciona mediante procedimientos establecidos de manera racional, sino que las acciones de política publica se deben a actores concretos, y están además determinadas por reglas informales y por aspiraciones o necesidades y experiencias personales de los participantes, que están en interacción constante con un entorno institucional (Friedberg, 1993). 
Esta manera de definir los procesos de gobierno a nivel local recupera la dimensión compleja, dinámica y conflictiva de las interacciones entre actores en una política pública, porque se aleja de la visión racional sostenida por la mayoría de los estudios de los asuntos públicos, los cuales plantean las políticas públicas como constructos sociales, producto de la planificación y el cálculo, y se ubican únicamente en los planos formal, legal y racional (Lasswell, 195I).

$\mathrm{Al}$ aplicar el concepto de acción organizada a los procesos de gobierno se reconoce que su funcionamiento es un asunto de buscar soluciones a las causas que determinan los problemas, las cuales requieren la implantación de acciones que contribuyen al logro de objetivos formales e intereses de los actores involucrados; los conceptos centrales del análisis son las acciones y sus actores, en donde se reconoce la existencia de la formalización y codificación de la regulación en un contexto institucional; que los miembros de las organizaciones están conscientes de ello, y de la existencia de un fin específico de la regulación (Friedberg, 1992).

Desde este enfoque conceptual se admite que en todo proceso de gobierno predomina un actor, donde los actores interactúan en un juego político de intereses en que hay perdedores y ganadores; este planteamiento es muy útil para explicar las políticas públicas locales en México, las cuales se caracterizan por la centralización de las decisiones en las agencias federales y estatales, la preeminencia de la racionalidad política y el carácter opaco de las decisiones de política pública.

La conceptualización de los procesos de gobierno como un sistema de acción concreto reconoce que su funcionamiento obedece parcialmente a sus características formales, y que los campos de acción están más estructurados de lo que aparentan; que son varios campos de acción los que se interrelacionan de manera compleja y conflictiva (Crozier y Friedberg, 1977). Esta postura se aleja de los análisis que solo describen las jerarquías, la coordinación, las reglas y los procedimientos, y ocultan la competencia, la negociación y el conflicto.

La consecuencia conceptual de adoptar un enfoque de esta naturaleza para el análisis de los procesos de gobierno consiste en que este se concibe como un constructo social que tiene objetivos cambiantes, en función del problema público que se busca resolver; en su funcionamiento no existe una manera única de resolverlo; se construyen redes de política pública con una constante entrada y salida de actores; hay una interrelación mutua con el entorno; sin embargo, cada organización pública logra mantener su identidad, especificidad y libertad (Weick, 1976).

En esta propuesta conceptual de análisis de los procesos de gobierno a nivel local se reconoce que las decisiones de política pública se toman con base en información imperfecta; es decir, los decisores no pueden conocer a la perfección todas las 
posibilidades de solución de un problema público; que en la definición y resolución de una problemática concreta participan no uno sino varios actores de diversas organizaciones públicas y de diferentes ámbitos de gobierno.

\section{Niveles de análisis de los procesos de gobierno a nivel local}

El análisis de los procesos de gobierno como resultado de un conjunto organizacional en interacción es necesario considerarlo como un constructo social complejo (Del Castillo, 1996), cuyo funcionamiento es consecuencia de las interacciones de sus actores; se pone en el centro al actor y sus acciones que en conjunto representan una política pública, entendida como una decisión técnica, política y de valores, lo cual es producto de:

I. un proceso múltiple, es decir, que en la definición del problema participan diversas actores y organizaciones con intereses, objetivos y metas diferentes;

2. un proceso ambiguo, porque cada actor define de manera subjetiva el problema e identifica sus soluciones, lo cual responde a su propia lógica de acción, pero que legitima tomando como referente su ubicación en la estructura funcional de la organización de la que forma parte;

3. un proceso continuo; lo que significa que la implantación de una decisión no soluciona los problemas públicos, sino que genera la necesidad de tomar nuevas decisiones, por lo que sus principales características son su inestabilidad y su constante cambio;

4. un proceso social, porque la subjetividad de los actores, ubicados en su entorno social específico, influye en su perspectiva de la problemática que se pretende solucionar, o al menos está presente en el discurso que justifica sus acciones;

5. un proceso político, porque la toma de decisiones es realizada con base en la estructura de poder que domina a los actores involucrados, en la cual uno de ellos logra imponer y negociar su propia lógica de acción.

El análisis de los procesos de gobierno debe hacerse bajo un esquema conceptual que considere cuatro elementos básicos: 1) el contexto; 2) las organizaciones públicas y sus actores; 3) las acciones, y 4) sus resultados, para lo cual, el estudio se centra en las interrelaciones de los actores a partir de la identificación de un problema público concreto.

El concepto de acción pública organizada aplicable a los procesos de gobierno parte de tres supuestos del funcionamiento de la administración pública local. Pri- 
mero, la existencia dentro de las organizaciones de actores con racionalidad limitada; segundo, las soluciones a los problemas públicos son contingentes, y tercero, el poder como capacidad de decisión de los actores. Estos supuestos perciben el funcionamiento del gobierno como un constructo político, es decir, resultado de la interacción de sus participantes, lograda por acuerdos, persuasiones o imposiciones construidos con base en relaciones de poder.

Para el caso de México, en el análisis de los procesos de gobierno es importante considerar el culto que se tiene al poder y el clientelismo como estrategia de política pública, ya que eso genera autoritarismo, centralismo y oportunismo, tanto en la toma de decisiones como en su implementación, sin lo cual no se podría explicar la estructura y el funcionamiento de las organizaciones públicas a nivel local.

Con fundamento en estos supuestos, los procesos de gobierno son resultado de intenciones, reflexiones, anticipaciones, cálculos y, en el caso mexicano, de la imposición o inducción en la definición de los problema públicos; por ello es necesario concebirlos como constructos sociales donde las normas y reglas establecidas, la historia personal y los procesos de aprendizaje de las normas y los valores sociales desempeñan un papel fundamental. Por esto, la racionalidad de los actores se halla en la orientación de sus acciones, en función de la naturaleza de la información y las representaciones que los actores tienen de los problemas y sus soluciones en un momento histórico determinado.

Para el análisis de los procesos de gobierno en México es útil tomar en cuenta la racionalidad limitada, concepto propuesto por March y Simon. Desde esta perspectiva conceptual, las soluciones a los problemas públicos son contingentes; es decir, están determinadas por eventos, dispositivos y, en buena parte, por la percepción de ellos en circunstancias específicas que se transforman en el tiempo, es decir, son dinámicas (March y Simon, 1958). Sin embargo, estas soluciones contingentes promueven sus propias reglas creadas por los actores, quienes incorporan en su comportamiento aspectos formales e informales de la organización de la que forman parte (Friedberg, 1992).

El análisis de los procesos de gobierno, al considerar la racionalidad limitada de los actores, no puede darse a partir de un modelo de construcción a priori, sino que es necesario reconstruir las relaciones, negociaciones y comportamientos de los actores, aspectos que pueden ser identificados en sus acciones. Estas interacciones son esencialmente constructos de poder, $y$ pueden ser percibidas y caracterizadas a partir de la información y los argumentos que justifican las acciones de política pública.

El concepto de sistema de acción organizada implica el análisis de interdependencia e intercambio negociado de acciones de los actores, producto de una regu- 
lación múltiple, es decir, de los mecanismos formales e informales. Esto conduce al reconocimiento de la complejidad dentro de los procesos de gobierno, que implica el reconocimiento de tres supuestos sobre las interacciones de los actores; primero, la racionalidad, que reconoce la existencia de información y conocimientos limitados; segundo, interdependencia dinámica entre los actores, y tercero, coexistencia de normas y valores particulares y universales (Crozier y Friedberg, 1977).

Por otro parte, el concepto de acción organizada recupera la existencia de un orden local, por medio del cual los actores establecen, al menos provisionalmente, sus acuerdos de negociación e interacción. Su estructuración implica la interdependencia y el intercambio negociado, donde el poder es su esencia y no un atributo; una capacidad de intercambio negociado de posibilidades de acción. En este contexto, los recursos de poder son dos, las posibilidades de acción y el manejo de zonas de incertidumbre; ambos se refieren a las posibilidades de los actores para actuar con libertad; por otra parte, la dinámica en las relaciones de poder está dada por una gran variedad de recursos, barreras, condiciones de intercambio y la facilidad de trasladar los costos a otros actores (Friedberg, 1992).

La regulación múltiple de los procesos de gobierno es producto de normas formales e informales provenientes, por un lado, de los objetivos, la estructura y las funciones formales de las organizaciones y, por el otro, de los intereses particulares, de las prácticas, interacciones y relaciones no previstas de los actores; es por ello que la decisión de política pública puede ser vista como producto de la negociación, imposición e inducción de los involucrados en la búsqueda de soluciones a los problemas públicos, donde la regulación formal jamás es total, sino que solo desempeña el papel de definir los espacios de negociación y de acción de los actores.

En resumen, el concepto de acción pública organizada es muy útil para explicar la dimensión organizacional de los procesos de gobierno; implica reflexionar sobre el concepto orgánico de la organización pública, sobre la complejidad del comportamiento de los actores involucrados en la toma de decisiones de política pública y el papel del contexto y el dinamismo en las estrategias de acción que buscan solucionar los problemas públicos, aspectos que se abordarán en los siguientes apartados.

\section{De la racionalidad a la complejidad en la toma de decisiones de política pública}

El estudio del comportamiento del individuo dentro de las organizaciones es un campo muy amplio, en el cual se abordan las dimensiones cognitiva y lingüística, 
espacio-temporal, psíquica y afectiva, simbólica, de la diferenciación con el otro y la psicopatológica (Chanlat, 1990). Las dimensiones del individuo, considerado en sus procesos de interacción, permiten interpretar la de los actores de política pública no de manera racional e impulsada solo por factores técnicos, sino por la influencia de múltiples factores de orden político, económico, social y hasta emocional.

Esta postura de la complejidad del comportamiento de los tomadores de decisiones en política pública deja de lado la conceptualización instrumental, de adaptación y de manipulación de los involucrados en los procesos de gobierno y propone rescatar su dimensión cualitativa. Esto significa alejarse de un enfoque dominado por las categorías racionales, y ubicarse en uno donde el individuo es considerado objeto y recurso de las organizaciones; donde las relaciones entre los hombres se subordinan a los aspectos materiales y predominan las formas de pensamiento de corto plazo.

El comportamiento complejo del actor organizacional privilegia los aspectos interpretativos que los miembros tienen de los problemas públicos y sus soluciones, la dimensión egoísta del comportamiento político, las exigencias de la solidaridad y los imperativos éticos, ya que ello influye en el comportamiento de los individuos dentro de las organizaciones públicas. Esta manera de definir los procesos de gobierno implica no encerrarlos en un mundo demasiado estrecho, ya que de hacerlo se reflejaría una imagen fragmentada de las políticas públicas a nivel local.

La perspectiva del comportamiento complejo de los actores en política pública requiere sustituir el concepto de los procesos de gobierno como sistema cerrado y autosuficiente, por uno abierto, que revalore las relaciones de poder dentro del gobierno, así como el carácter abierto del gobierno con su entorno, respecto del cual toda decisión en política pública se orienta y centra en una parte específica.

Cada actor es un ser humano complejo, según Chanlat (1994), un ser genérico y singular, activo y reflexivo, de palabra, de deseo y pulsión, simbólico, y responde a un espacio y a un momento histórico específico. Un análisis del comportamiento de los actores en los procesos de gobierno a nivel local desde la perspectiva de la complejidad requiere poner en el centro del estudio los argumentos del discurso de los actores de política pública, en el cual influyen factores formales, informales y de valores.

Esta es una visión subjetiva e interactiva que representa el retorno del actor a la escena del análisis de las políticas públicas, desde donde se podrá comprender el sentido otorgado a sus acciones, y para lo cual el método etnográfico, la entrevista a profundidad, las historias de vida, el análisis del discurso y las decodificaciones simbólicas son las principales técnicas de estudio del comportamiento de los procesos de gobierno. 
El rescate, en el análisis de las políticas públicas, de la dimensión compleja del comportamiento organizacional permite combinar, en el estudio, los aspectos formales e informales de su funcionamiento organizativo. La organización formal es el conjunto de límites, cadenas jerárquicas y procedimientos de coordinación; por su parte, la organización informal no es más que la existencia de factores que engloban y sobrepasan el marco formal restrictivo de las organizaciones.

Introducir la dimensión de la complejidad del comportamiento de los actores implica percibir los procesos de gobierno como un problema en términos de Crozier (1977), en el cual las soluciones y acciones de los actores son producto de su margen de libertad, de la forma de definir el problema, del análisis de las dificultades en la aplicación de las soluciones identificadas, del uso de las normas de la organización y de una imposición negociada de una forma de definir los problemas públicos.

Desde esta perspectiva, los aspectos racionales y subjetivos de los procesos de gobierno representan el fundamento de las acciones de los actores, ya que son la base del conjunto de relaciones establecidas y donde se materializa la complejidad del comportamiento de los actores dentro de las organizaciones. Dichas acciones se construyen por relaciones de poder, que representan la posibilidad individual de actuar sobre los otros actores, materializada en la capacidad de decisión de los actores, por lo que el poder es una relación y no un atributo de los actores (Crozier, 1977).

Desde este enfoque de los procesos de gobierno, las acciones de política pública son resultado del enfrentamiento entre racionalidades contingentes, múltiples y divergentes de actores relativamente libres que utilizan los recursos de poder de que disponen; donde cada actor otorga un nivel jerárquico diferente a los objetivos de política pública, por lo que su existencia está condicionada por la estructura de poder dominante y no como una consecuencia lógica de leyes y normas. Es por ello que las relaciones de poder entre los actores son el principal instrumento de construcción de las políticas públicas, mecanismo que permite la integración entre estructura, función y actores en los procesos de gobierno; además, es un instrumento que permite reglamentar la cooperación y que está ligado a las pautas de comportamiento de la sociedad y a la capacidad de los actores.

Otro aspecto importante a considerar es que los actores involucrados en las políticas públicas locales pocas veces tienen objetivos claros, proyectos coherentes y estables; sus comportamientos son restringidos y limitados según su entorno organizacional y el momento histórico específico, por lo que el concepto de acción pública organizada conduce a buscar la racionalidad de los actores en el entorno y no dentro de las organizaciones públicas; en el análisis de los procesos de gobierno 
es importante reconstruir las relaciones de poder de los actores a partir de los argumentos y datos del discurso que utilizan para definir problemas públicos.

\section{Las políticas públicas como resultado del entorno institucional}

Las políticas públicas como constructos sociales complejos no existen en abstracto, sino que se generan en un entorno institucional, que se refiere a las reglas formales e informales que de manera directa o indirecta determinan su diseño, operación y resultados. El entorno puede ser clasificado en dos dimensiones: una general, constituida por un conjunto de condiciones semejantes para todas las organizaciones públicas; otra particular o inmediata, de la cual cada organización y actor extrae sus principales pautas de comportamiento y hacia la que se orientan las acciones de política pública.

Considerar la intervención de diversos actores en el diseño, implementación y evaluación de las políticas públicas supone que son producto de acuerdos e imposiciones basadas en las relaciones de poder resultantes de reglas formales e informales, que se desarrollan en un sistema más amplio y complejo de actores y determinantes institucionales, lo cual significa que el contexto limita pero no elimina la libertad del actor para tomar decisiones. En este sentido, las decisiones de política pública se ubican en un contexto particular que no es solo un dato objetivo, sino un conjunto de variables que los actores deben reinterpretar para darles un peso, prioridad y sentido particular en cada situación de decisión, por lo que estos también contribuyen a las transformaciones del entorno que determina los procesos de gobierno

Las relaciones interdependientes entre los actores de política pública y su entorno no son equilibradas, sino inclinadas en favor de algunos de ellos, quienes buscan persuadir o imponer sus comportamientos de la manera más ventajosa, buscando resolver problemas pero también cumplir intereses personales. Por esta razón, las acciones de los actores son sistemas que cumplen también una función social, es decir, atienden una o varias demandas de la sociedad, por lo que su efecto va más allá del sistema interno de la organización pública de la que surgieron, más aún si se trata de resolver problemas públicos.

Las interferencias del entorno en las políticas públicas son numerosas y constantes, por lo que buscarán controlar su contexto por medio de negociaciones, acuerdos o imposiciones con actores de otras organizaciones. Estos escenarios de interacción se establecen mediante relaciones de poder (Friedberg, 1993); situación en la que los 
actores cumplen una doble función: representan a la organización en el entorno y, a la vez, son representantes del contexto en la organización.

Desde este punto de vista, el entorno institucional es heterogéneo, no unificado, sino diversificado, fraccionado y segmentado, por lo cual debe desarrollarse una estrategia diferenciada de política pública, cada una de las cuales debe responder a un problema público concreto, por lo que tendrá orientaciones particulares y estructuras específicas; con esto se reconoce que en una política pública no hay una, sino un conjunto de acciones, que representan un sistema de acción organizada.

Reconocer que en el diseño, la implantación y evaluación de toda política pública participan diversas organizaciones y actores, significa considerar que cada una de ellas desarrolla su propia lógica de acción y, por lo tanto, de interacción, lo que implica la existencia de una influencia recíproca entre las organizaciones públicas y su entorno. El conjunto organizacional es atravesado así por su entorno social, el cual estructura de manera más o menos directa y visible los procesos de gobierno. Por ello, cada uno de los actores se sitúa en un contexto institucional particular que, consciente o inconscientemente, utiliza para tomar decisiones de política pública.

En virtud de que los actores de política pública en las organizaciones tienen cierto margen de libertad y de que los factores institucionales del entorno son incorporados en función de su capacidad de acción y de negociación, la relación organización-entorno no es un proceso de adaptación, sino de interacción, por lo que su análisis está condicionado por cómo y dentro de qué límites la lógica de las relaciones de los actores incorpora las influencias del contexto en los procesos de gobierno.

Esta propuesta interpretativa reconoce que en toda política pública, para cumplir con sus objetivos, los actores deben negociar con los principales actores de su entorno, por lo que se debe caracterizar el tipo de relación existente entre ellos y el momento histórico en el que se establecen las interacciones que responden a una problemática identificada en el espacio y en el tiempo, por lo que estas se transforman en el tiempo en función del entorno institucional, de la situación social y de los actores involucrados, por lo que surge el concepto de cambio como una variable más para explicar los procesos de gobierno a nivel local.

\section{Contingencia de la política pública: el funcionamiento cíclico de los procesos de gobierno}

El concepto de cambio ha sido definido de diversas maneras en las distintas corrientes teóricas de los Eo. Por ejemplo, las teorías de la acción racional, entre las que se 
encuentra la teoría de la burocracia, la organización científica del trabajo y la escuela de las relaciones humanas, plantean que la acción organizada es una acto consciente, premeditado y guiado por una intención o un propósito; por ello expresan que las acciones del actor están generalmente medidas por su conocimiento de la realidad social hacia la que están orientadas. En este enfoque, la organización es vista como un conjunto de diversos actores que persiguen fines coherentes, que emplean los medios más apropiados para alcanzar los objetivos y metas; por lo que para que se genere un cambio, el comportamiento de sus miembros será el instrumento de transformación (Coronilla y Del Castillo, 2000).

Por su parte, la corriente teórica de la conducta racional adaptativa, planteada por el enfoque de la contingencia, percibe la organización como una totalidad racional que actúa de manera previsora y prospectiva para alcanzar sus objetivos, los cuales generalmente responden a las transformaciones del entorno (Coronilla y Del Castillo, 2000). Desde esta perspectiva, en las organizaciones los procesos de funcionamiento son ordenados por objetivos y acciones definidas en el tiempo y en el espacio, establecidos en estructuras funcionales, dotados de fines e instrumentos de previsión y control.

Las posibilidades de cambio son diversas, van desde la conservación, la adaptación, la reelaboración y la transformación de las estructuras organizacionales, adoptadas para dar respuesta a las transformaciones del entorno. Otras veces, los cambios surgen como reacción a futuras presiones del entorno, por lo que el reto es adoptar formas de organización que permitan rapidez y flexibilidad en la solución de la problemática que presenta el contexto; por ello, la organización debe tener capacidad para transformar sus estructuras y procesos de trabajo. En este enfoque el elemento central de cambio es la estructura funcional (Coronilla y del Castillo, 2000).

Por su parte, desde la perspectiva de la acción organizada se plantea la existencia heterogénea y múltiple de actores y de una diversidad de objetivos por cumplir, en un entorno de diversas organizaciones en interacción, por lo que sus actores no necesariamente tienen objetivos claros y proyectos coherentes, sino que podrían cambiar a la mitad del camino transformando sus percepciones o sus metas y maneras de actuar. Es por ello que el cambio parte del supuesto de que las acciones son producto de la interacción de un conjunto organizacional y una respuesta a un problema en un momento histórico determinado. Esto significa que el cambio está siempre presente; se materializa en la entrada y salida de actores involucrados en las políticas públicas $y$ en el uso de acciones que en un momento determinado fueron rechazadas por ser consideradas inviables, o bien en la adopción de otras que incluyen elementos nuevos que no se habían considerado (Crozier y Friedberg, 1977). 
El cambio, por consiguiente, tiene dos facetas: en una actividad, en una función, modo de operación o bien en una técnica; y el cambio de las características y los modos de regulación de un sistema organizado, incluso de una transformación de las estructuras y los juegos de poder. El cambio se manifiesta mediante un proceso de aprendizaje que implica el descubrimiento, la creación y la adquisición, por los actores involucrados, de nuevas formas de relación, nuevos modos de razonamiento, es decir, de nuevas capacidades de negociación (Crozier y Friedberg, 1977).

Si bien es cierto que en los procesos de gobierno a nivel local, las políticas públicas surgen como respuesta a una situación social problematizada, son producto contingente de las interacciones de diversos actores, por lo que su lógica no es el aprovechamiento óptimo ni el máximo rendimiento de los recursos, sino encontrar la solución satisfactoria para los actores involucrados, cuya racionalidad y legitimidad se encuentra en su contexto. Por ello, la naturaleza más profunda de las políticas públicas es su alta dinamicidad, lo que implica la transformación constante en la forma de definir los problemas públicos.

Esto significa que en los procesos de gobierno se desarrollan diversas estrategias, técnicas, tareas y lógicas de acción en ciertos periodos y espacios de las organizaciones públicas, por lo que el cambio no necesariamente abarca todo el conjunto organizacional del gobierno local, sino que es parcial a un espacio organizado concreto vinculado a un problema público y responde a los intereses dominantes en el sistema político local.

En esta propuesta conceptual, el funcionamiento cíclico de los procesos de gobierno no significa ni una etapa lógica del desarrollo de la administración pública local ni la imposición de un nuevo modelo organizacional, ni la transformación de un sistema organizacional local, sino que es contingente a las políticas públicas al reconocer que los actores no están atados a sus tareas y percepciones de manera pasiva y limitada, sino que están dispuestos a cambiar si encuentran interés en nuevos objetivos y relaciones de interacción con su entorno.

En el análisis de la acción pública organizada en los procesos de gobierno a nivel local, el cambio es una fuente explicativa relevante para comprender la institucionalización de las políticas públicas, ya que ahí repercute la naturaleza de acciones implantadas, las cuales son respuestas provisionales a la problemática dominante en la agenda pública en un momento histórico específico. Para intentar una explicación en los procesos de gobierno, el concepto de estrategia representa el instrumento conceptual y metodológico más adecuado para reconstruir las soluciones de los problemas públicos en los gobiernos locales. 


\section{Las políticas públicas locales como un constructo estratégico}

Para analizar los procesos de gobierno desde una perspectiva de la estrategia se requiere considerar dos aspectos básicos: primero, es necesario entender la naturaleza y las manifestaciones de las políticas públicas y, segundo, comprender la manera como estas se construyen; por ello, en los siguientes apartados la estrategia se abordará como constructora de procesos de solución de problemas públicos, y se definirá la estrategia como un proceso de interacción discursiva, ya que son estos los que establecen los acuerdos e imponen las acciones entre los actores involucrados en la búsqueda de la solución a los problemas públicos locales.

\section{Concepto y naturaleza de estrategia}

El análisis de la acción pública organizada para reconstruir las estrategias de solución a los problemas públicos parte de cuatro supuestos básicos sobre la naturaleza de la relación entre estrategia y funcionamiento de las organizaciones (Crozier y Friedberg, 1977):

I. La estrategia no es la predicción del futuro de los problemas públicos, sino el establecimiento de una direccionalidad de sus acciones respecto de un entorno social específico y un momento histórico determinado.

2. La estrategia no concibe las acciones de los procesos de gobierno como totales, racionales y lineales, sino como móviles y cambiantes, en donde el poder, la cooperación y el conflicto son sus principales determinantes institucionales, aunque no los únicos.

3. Los actores de política pública involucrados en el diseño e implantación de una estrategia no controlan del todo a los demás actores, pero sí pueden dirigirlos hacia la cooperación mediante la negociación, persuasión o imposición en una relación de poder asimétrica.

4. La estrategia es un marco conceptual y metodológico para entender y explicar la estructura y el funcionamiento de los procesos de gobierno a nivel local como producto de un conjunto organizacional.

La estrategia es entendida como el conjunto de percepciones, acciones y orientaciones establecidas en una organización para alcanzar un conjunto de objetivos. Este concepto ayuda a explicar a los procesos de gobierno como un conjunto de diversas lógicas de acción. Por ello, las estrategias son producto de un proceso múltiple, 
ambiguo, continuo, social y político, por lo que los actores, al desempeñarse en esa complejidad de relaciones, encuentran sus márgenes de libertad. Por esta razón, desde la perspectiva de la acción pública organizada, lo sustantivo en los procesos de gobierno no es controlable por un solo actor, sino por varios, que provienen de diversas organizaciones públicas.

Un enfoque múltiple de la estrategia se refiere a que en la definición del problema de política pública y en la búsqueda de soluciones participan diversos actores de organizaciones públicas, aunque en el caso mexicano una de ellas centraliza la toma de decisiones. Como resultado de un proceso ambiguo significa que la información sobre las que se toman las decisiones y se define la problemática siempre es insuficiente para llegar a una solución óptima, por lo que se busca solo la más satisfactoria para los actores involucrados y para el momento histórico en que se encuentra el asunto público en cuestión.

La estrategia de política pública es inestable, es un proceso en constante cambio, es decir, no es una solución óptima, sino que su implantación es de carácter incremental; además, las acciones implantadas son generadoras de nuevas decisiones, que requieren nuevas acciones. Es social, porque la estrategia responde a un contexto institucional específico hacia el cual están orientadas las acciones, por lo que se ve influida por las percepciones de los actores del entorno. Lo político se halla en la construcción de la estrategia, ya que es producto de relaciones de poder, es decir, responde a la lógica de acción de actores que cuentan con mayores recursos de decisión.

Definida la estrategia de política pública como resultado de un proceso múltiple, ambiguo, continuo, social y político, los procesos de gobierno pueden verse como una interacción de acuerdos y desacuerdos entre los diversos actores que la integran, los cuales son resueltos mediante la negociación e imposición de cierta percepción de los problemas públicos por uno o varios actores con poder de decisión; cuando se llega a compartir la definición del problema y las acciones para hacerle frente se institucionalizan, y se han establecido mecanismos de interacción entre los actores, es cuando surge el sistema de acción pública organizada.

En el análisis de la acción pública organizada lo esencial es encontrar la direccionalidad de las acciones de política pública, caracterizando la naturaleza de los acuerdos, desacuerdos e imposiciones. En este sentido, la acción pública organizada es la forma de combinar percepciones, acciones y lógica de orientación de cada uno de los actores involucrados, los cuales para ser considerados actores necesitan formar parte de una organización pública, tener atribuciones y recursos de decisión y acción, por lo cual pueden participar en la búsqueda de soluciones a situaciones socialmente problematizadas. 
Como se dijo anteriormente, para el estudio de la acción pública organizada se requiere analizar al actor, sus acciones y sus instituciones. Adquiere su materialidad como decisión y acción, entendidos como elementos constructores de un espacio organizado. Por esta razón, la organización no es la codificación y estructuración del comportamiento de los actores, sino la delimitación de los espacios de acción para tomar decisiones y llevar a cabo las acciones; por lo tanto, la organización es generadora de pautas y valores de comportamiento a partir de los cuales se construye la lógica de los involucrados en los procesos de gobierno. La decisión y la acción de los actores representan su postura frente a los problemas públicos, con lo cual se construye la direccionalidad de la estrategia de política pública.

Las acciones de política pública de los actores del sistema de acción pública organizada se imponen a los demás actores mediante las relaciones y los recursos de poder; encuentran en los argumentos del discurso que sustentan la definición de los problemas públicos; es además el principal espacio de interacción entre actores que provienen de diferentes organizaciones, motivo por el cual es necesario conceptualizar la relación entre organización, poder y discurso en los procesos de gobierno en el ámbito local.

\section{Acción pública organizada como interfaz de interacción en los procesos de gobierno}

Para que se cumplan los supuestos de que la estrategia de política pública es constructora del sistema de acción pública organizada, de que la estrategia es producto de relaciones de poder que se establecen entre diversos actores en una interfaz organizacional, que la estrategia requiere la interacción de los actores de diversas organizaciones públicas, es necesario caracterizarlas como interacciones discursivas, las cuales se generan entre los actores involucrados en la solución de los problemas públicos.

El punto de partida y llegada del análisis de la interacción entre los actores de un sistema de acción pública organizada a partir de los argumentos de discurso radica en los aspectos formales de las organizaciones públicas - estructura, normas, funciones, procesos de trabajo-, lo cual es necesario para comprender el tipo de interacción establecida entre los actores, ya que es en esta parte de la organización donde los actores legitiman los argumentos discursivos que justifican sus percepciones, decisiones y acciones, es decir, los actores argumentan en la organización formal sus posturas para ocultar su parte subjetiva. 
Los actores expresan su postura ante los problemas públicos a partir de tres aspectos centrales; primero, el para qué, es decir, hacia dónde se deben orientar las decisiones y acciones de una política pública; segundo, el qué, que consiste en la descripción específica de las causas que generan un problemas público y, tercero, cómo se llevan a cabo las acciones, es decir, la estrategia a seguir en el corto, mediano y largo plazo (Majone, 1989).

$\mathrm{Al}$ establecer la relación entre estrategia, estructura de poder y argumentos del discurso se requiere conceptualizarlo como un proceso que va más allá de la simple transmisión e intercambio de mensajes y recursos, y considerarlo como un proceso de interacción que contribuye al establecimiento de un orden y sentido de las acciones, por lo que se pueden identificar tres dimensiones del discurso: primero, como una práctica social de interacción entre los actores por medio de la cual se establecen las relaciones de poder; segundo, como un proceso generador de acuerdos y desacuerdos, por medio de los cuales se establecen estrategias; tercero, un proceso generador de interpretaciones distintas en cada actor involucrado (Taylor, 1988).

Un enfoque conceptual de esta índole sobre el discurso en las organizaciones públicas conduce a identificar la dinámica organizacional como un asunto de la palabra (Chanlat, 1990); es decir, que los actores de política pública en la mayor parte de su tiempo intercambian información, recursos e interpretaciones, por lo que sus argumentos se convierten en el instrumento metodológico para la reconstrucción de los procesos de gobernar a nivel local.

El análisis del discurso en el campo de las ciencias sociales es una técnica compleja (Reygadas, 1999) cuyo modelo analítico comprende tres aspectos fundamentales: el tipo de discurso, sus condiciones de producción, recepción y circulación, y el funcionamiento del discurso del poder y la ideología (Haidar, 1998).

Según Reygadas los criterios de clasificación para identificar el tipo de discurso son: el objeto discursivo (Foucault, 1989), las funciones discursivas (Jakobson, 1980), el sujeto del discurso (Pêcheux, 1969), el aparato ideológico en el que se genera el discurso (Gramsci, 1975; Althusser, 1985; 1987), la macroperación discursiva, formalidad versus informalidad y oralidad versus escritura (Ong, 1987).

Las condiciones de producción, recepción y circulación del discurso giran en torno a sus condiciones de posibilidad (Foucault, 1980), la articulación entre la formación social, la formación ideológica y la formación discursiva del sujeto generador del discurso (Pêcheux, 1971; Althusser, 1985, 1987), las formaciones imaginarias (Pêcheux, 1969), la relación discurso-coyuntura (Robin, 1976), la gramática de producción y recepción (Veron, 1973), la aceptabilidad y la topología del discurso (Faye, 1978), los procesos de interdiscursividad, la situación comunicativa (Hymes, 1964). 
Por su parte, el discurso del poder y la ideología se desarrolla en función del objeto discursivo prohibido (Foucault, 1989), el objeto discursivo impuesto (Foucault, 1989), la interdiscursividad (Plantin, 1996), las formaciones imaginarias (Pêcheux, 1969), la situación personal, espacial y temporal (Benveniste, 1986), los actos del discurso (Ducrot, 1980), los argumentos (Plantin, 1996), las estrategias de persuasión (Perelman, 1989), las esquematizaciones del objeto discursivo (Grize, I996), los lugares comunes, prototipos, estereotipos y clichés (Thompson, I993), los procesos de repetición, de énfasis y redundancia, las relaciones entre lo implícito y lo explícito (Perelman, 1989) y los tropos (Lakoff, 1986).

En resumen, el análisis de los procesos de gobierno como un sistema de acción pública organizada como producto de la interacción con base en argumentos discursivos de los actores involucrados en la solución de los problemas públicos supone que cada actor genera tres tipos de discurso: uno general, que comparten todos los actores del sistema; uno organizacional, que es reflejo de cada organización pública; y uno particular, que justifica las soluciones de política pública a los problemas públicos.

Los argumentos del discurso se construyen en función de los aspectos formales e informales de las organizaciones públicas, de la formación social de los actores y las condiciones institucionales de producción y reproducción del discurso, elementos a partir de los cuales hay que construir el modelo operativo de análisis del discurso de los actores organizacionales, sobre cuya base se pueda hacer la reconstrucción de los procesos de gobierno en el ámbito local, a partir de lo cual es posible caracterizar las estrategias de política pública en los municipios mexicano.

\section{A manera de conclusión}

El análisis de los procesos de gobierno en el ámbito local como un sistema de acción pública organizada es un marco conceptual por aplicar en la realidad local mexicana, a partir de lo cual se recuperará el concepto de complejidad del gobierno, identificado como un conjunto organizacional. Significa dejar de concebirlo como un ente monolítico, homogéneo y aislado, y considerarlo como un conjunto de organizaciones en interacción conflictiva para diseñar soluciones de políticas públicas, cada una en su propia estructura y lógica de funcionamiento, y las políticas públicas como resultado de un proceso de gobierno múltiple, ambiguo, continuo, social y político.

Una postura conceptual que recupera el sistema de acción pública organizada como variable explicativa del funcionamiento de los procesos de gobierno significa transitar: 1) del estudio de la organización al de un conjunto de organizaciones en 
interacción; 2) de considerar objeto de estudio la parte formal y codificada de las agencias, al de los actores, sus estrategias de acción y su entorno institucional; 3) del reconocimiento de actor racional al de un actor complejo; 4) de la identificación de que el gobierno funciona con base en la adaptación al entorno al de una relación de interdependencia; 5) de las estrategias consideradas estables a la conceptuación de estas como altamente contingentes; 6) que la estrategia de acción es constructora del sistema de acción pública organizada, y 7) que las estrategias se construyen por relaciones de poder mediante una interacción discursiva basada en argumentos.

\section{Bibliografía}

Arellano, D., E. Cabrero y A. del Castillo

2000 Reformando al gobierno, una visión organizacional del cambio gubernamental, Miguel Angel Porrúa/cide, México.

Benson J. K.

I975 "The interorganizational network as a political economy", Administrative Science Quarterly, vol. xx, pp. 229-249.

Benveniste, Emile I980 Problemas de lingüistica general, Siglo xxi Editores, México.

Blau P. M. y W. R. Scott

I963 Formal organization: a comparative approach, Routledge y Kegan Paul, Londres.

Boudon, R.

I979 La logique du social: introducción à l’analyse sociologique, PUF, París.

Burns, T. Y. y G. M. Stalker

196I The management of innivation, Tavistock, Londres.

Cabrero, Enrique

2005 Acción pública y desarrollo local, FCE, México.

Castillo, Arturo del

1996 Ambigüedad y decisión: una revisión a la teoría de las anarquias organizadas, CIDE, México [documento de trabajo 36].

Chanlat, J. F.

I990 L'individu dans l’organisation, les dimensions oubliées, Éditions Eska, Canadá.

I994 "Hacia una antropología de la organización", Gestión y política pública, III(2), pp. 3I7-364. 
Coronilla, Raúl y Arturo del Castillo

2000 "El cambio organizacional: enfoques, conceptos y controversias", en D. Arellano, E. Cabrero y A. del Castillo, Reformando al gobierno, una visión organizacional del cambio gubernamental, Miguel Angel Porrúa/ CIDE, México, pp. 77-I36.

Crozier, M.y E. Friedberg 1977 L'acteur et le systéme, Editions du Seuil, París.

Dahl, R. A.

1957 "The concept of power", Behavioral Sciences, II, pp. 20I-215

Ducrot, Oswald

1980 Les mots du discours, Minuit, París.

Faye, Jean Pierre

I976 La critica del lenguaje y su economía, Alberto Corazón, Madrid.

Foucault, Michel

I980 El orden del discurso, Tusquets, Barcelona.

1989 Las palabras y las cosas, Siglo xxi Editores, México.

Friedberg, E.

1993 Le Pouvoir et la règle. Dynamiques de l’action organisée, Éditions du Seuil, París.

Grize, Jean Blaise

1996 Logique naturelle et communications, PUF, París.

Haidar, Julieta

1998 "Análisis de discurso", en J. Galindo Cáceres (comp.), Técnicas de investigación en sociedad, cultura y comunicación, Conaculta/Addison Wesley Longman, México.

Hall, D. T.

1963 "The concept of Bureaucracy: an empirical assessment", American Journalof Sociology, 49, pp. 32-40

Hickson, D. J.

1969 "Operation technology and organization structure: an empirical reaHymes, Dell H. ppraisal", Administrative Science Quarterty, xvi, pp. 378-397.

I964 Language in culture and society: a reader in linguistics and anthropology; Harper y Row, Nueva York.

Jablin F. et al.

1987 Handbook of organizational communication, Sage Publication, Londres. 
Jakobson, Roman

I980 El Marco del lenguaje; FCE, México.

Lakoff, George y Mark Jonson

I986 Metáforas de la vida cotidiana, Cátedra, Madrid

Lawrence, P. R. y J. W. Lorsch

I967 Adapter les structures de l'entreprise, D'organisation, París.

Luhmann, N.

I964 Function and formal organization, Duncker y Humblot, Berlín.

Majone, Giandomenico

I989 Evidencia, argumentación y persuasión en la formulación de políticas, Colegio Nacional de Ciencias Políticas y Administración Pública/FCE, México.

March, J. G. y J. P. Olsen

1989 Rediscovering institutions: the organizations basis of politics, The Free Press, Nueva York.

March, J. G. y H. A. Simon

I958 Teoría de la organización, editorial Ariel, España.

Merino, Mauricio

2013 Políticas Públicas. Ensayo sobre la intervención del Estado en la solución de problemas públicos, CIDE, México

Mouzelis, N. P.

1967 Organización y burocracia, Península, España.

Ong, Walter J.

1987 Oralidad y escritura — tecnologías de la palabra—, FCE, México.

Pecheux, Michel

1969 Hacia el análisis automático del discurso, Gredos, Madrid.

I97I "La sémantique et la coupure saussurienne: langue, langage, discours", Langages, 6 .

Perelman y Olbrechts-Tyteca

1989 Tratado de la argumentación, Gredos, Madrid, 1958.

Pfeffer J.

198I Power in organizations, Pittman, Boston.

Platin, Christian

1996 L'argumentation, Seuil, París.

Putnam L. et al.

1996 “Mtaphors of communication and organization”, en S. Clegg, C. Hara y W. Nord, Handbook of organization studfies, Sage Publication, Londres. 
Reygadas, Pedro

I998 "Argumentation: de la guerre a la paix Chiapas 1994", tesis del diplomado en estudios a profundidad en ciencias del lenguaje, Lyon.

Robin, Régine

1976 "Discours politique et conjoncture", en L’analyse du discours, Centre Educatif et Culturel, Montreal.

Thompson, J. B.

1993 Ideología y cultura moderna-teoría crítica social en la era de la comunicación de masas, UAM-X, México.

Tylor J.

1988 Une organisation n'est qu'un tissu de communication, Université de Montréal, Montreal.

Veron, Eliseo

1973 "Ideología y comunicación de masas. La semantización de la violencia y política”, Lenguaje y comunicación social, Nueva Visión, Buenos Aires.

Weick, Karl E.

1976 "Educational Organizations as Loosely Coupled Systems", Administrative Science Quarterly, 2I(I), marzo, pp. I-I9.

Woodward, J.

1958 Management and tebnology, HMso, Londres. 